Article

\title{
Steroidal Constituents from Roots and Rhizomes of Smilacina japonica
}

\author{
Yuwen Cui ${ }^{1,2,+}$, Xinjie Yang ${ }^{3,+}$, Dongdong Zhang ${ }^{4}$, Yuze Li ${ }^{5}$, Li Zhang ${ }^{3}$, Bei Song ${ }^{5}$, \\ Zhenggang Yue ${ }^{3}$, Xiaomei Song ${ }^{3}$ and Haifeng Tang ${ }^{1, *}$ \\ 1 Institute of Materia Medica, School of Pharmacy, Fourth Military Medical University, Xi'an 710032, China; \\ polaris_101025@163.com \\ 2 Department of Pharmacy, Xi'an Medical University, Xi'an 710021, China \\ 3 Shaanxi Collaborative Innovation Center of Chinese Medicinal Resource Industrialization, \\ Laboratory of New Drugs and Chinese Medicine Foundation Research, Shaanxi Rheumatism and Tumor \\ Center of TCM Engineering Technology Research, School of Pharmacy, Shaanxi University of Chinese \\ Medicine, Xianyang 712046, China; xxx211xxx@126.com (X.Y.); zhangl123666@163.com (L.Z.); \\ liuxingjian1981@163.com (Z.Y.); songxiaom@126.com (X.S.) \\ 4 The College of Pharmacy, Shanghai University of Traditional Chinese Medicine, Shanghai 200001, China; \\ zhangnatprod@163.com \\ 5 The College of Life Sciences, Northwest University, Xi'an 710069, China; lyz1990yeah@163.com (Y.L.); \\ songbei168@126.com (B.S.) \\ * Correspondence: tanghf71@fmmu.edu.cn; Tel.: +86-29-8477-4748 \\ + The authors contribute equally to this work.
}

Received: 6 March 2018; Accepted: 28 March 2018; Published: 30 March 2018

Abstract: Four new steroidal constituents (1-4) along with two known steroidal glycosides (5 and 6) were isolated from the roots and rhizomes of Smilacina japonica. Analysis of their physicochemical properties and spectroscopic profiles identified the compounds as (25S)- $5 \alpha$-spirostan-9(11)-en-3 $\beta, 17 \alpha$-diol (1); (25S)-5 $\alpha$-spirostan-9(11)-en- $3 \beta, 12 \beta$-diol (2); (25S)-5 $\alpha$-spirostan-9(11)-en-3 $\beta, 17 \alpha$-diol-3-O- $\beta$-D-glucopyranoside (3); (25S)- $5 \alpha$-spirostan-9(11)-en-3 $\beta$, $17 \alpha$-diol-3-O- $\beta$-D-glucopyranosyl-( $1 \rightarrow 2)$-[ $\beta$-D-glucopyranosyl-( $1 \rightarrow 3)]-\beta$-D-galactopyranoside (4); japonicoside $B(5)$; and japonicoside $C(6)$. All six compounds showed cytotoxic activity against SMMC-7712, Bel-7402, A549, H460, and K562 human cancer cells.

Keywords: Smilacina japonica; steroidal constituents; structure identification; cytotoxicity

\section{Introduction}

Smilacina japonica A. Gray is a perennial herb of the genus Smilacina (Liliaceae) that is mainly distributed in Asia, America, and Europe. As one of about 16 Smilacina species in China, S. japonica is widely distributed in Hebei, Shanxi, Shaanxi, Gansu, and Henan Provinces and is used in traditional Chinese medicine or consumed as a vegetable. Its roots and rhizomes are known to dispel pathogenic wind, remove dampness, promote blood circulation, and alleviate pain, and have long been used in folk medicine for the treatment of rheumatism, nervous headache, mastitis, carbuncle, and bruises [1-5]. Previous investigations of the bioactive compounds of S. japonica revealed four steroidal saponins [6-8]. As part of our exploration of the diversity of bioactive compounds in medicinal herbs growing in the Qinba Mountains [9-15], we investigated the chemical constituents of S. japonica in the present study. We identified six steroidal constituents (Figure 1), characterized their structure and pharmacological profiles, and evaluated their cytotoxicity against various human cancer cell lines. 


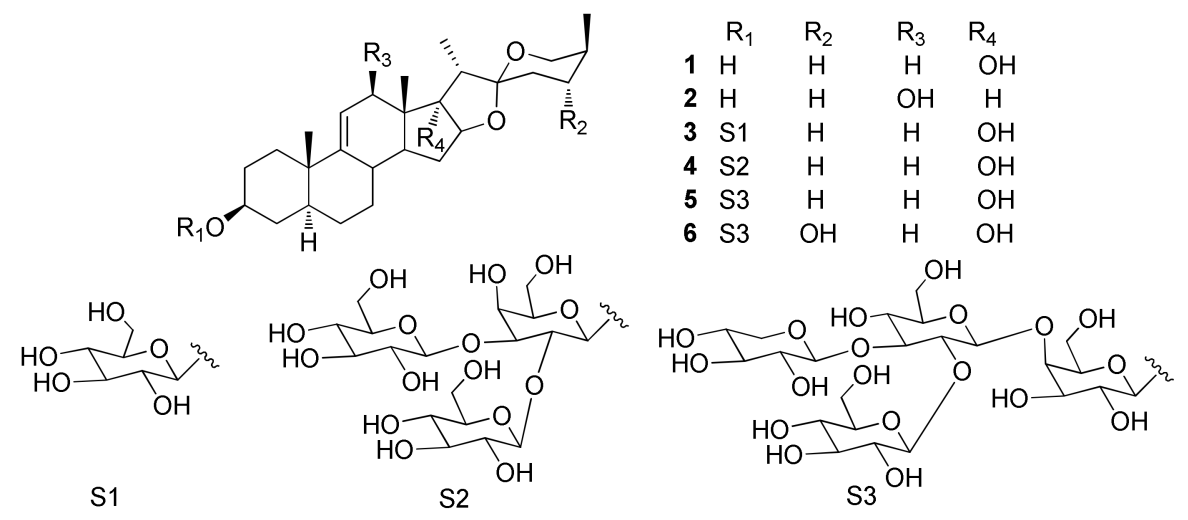

Figure 1. Chemical structures of compounds 1-6.

\section{Results and Discussion}

Compound $\mathbf{1}$ was isolated as a white, amorphous powder and was positive to Liebermann-Burchard and Molisch chemical reactions. The HR-ESI-MS analysis revealed a positive molecular ion peak at $m / z 431.3154[\mathrm{M}+\mathrm{H}]^{+}$, corresponding to the molecular formula $\mathrm{C}_{27} \mathrm{H}_{42} \mathrm{O}_{4}$ (calculated for $431.3161\left[\mathrm{M}+\mathrm{H}^{+}\right.$). The proton ${ }^{1} \mathrm{H}-\mathrm{NMR}$ spectrum of 1 showed the presence of four methyl protons at $\delta_{\mathrm{H}} 0.92(3 \mathrm{H}, \mathrm{s}, \mathrm{H}-18), 0.98(3 \mathrm{H}, \mathrm{s}, \mathrm{H}-19), 1.21(3 \mathrm{H}, \mathrm{d}, J=7.1 \mathrm{~Hz}, \mathrm{H}-21)$, and $1.05(3 \mathrm{H}, \mathrm{d}, J=7.1 \mathrm{~Hz}, \mathrm{H}-27)$ and an exocyclic olefinic proton at $\delta_{\mathrm{H}} 5.51(1 \mathrm{H}, \mathrm{d}, J=5.3 \mathrm{~Hz}, \mathrm{H}-11)$. The ${ }^{13} \mathrm{C}$-NMR spectrum showed 27 carbon signals corresponding to one double-bond carbon at $\delta_{\mathrm{C}} 145.5(\mathrm{C}-9)$ and 116.2 (C-11) and four methyl groups at $\delta_{\mathrm{C}} 16.4$ (C-18), $17.4(\mathrm{C}-19), 8.2$ (C-21), and 15.4 (C-27). The quaternary carbon signal at $\delta_{\mathrm{C}} 110.5$ was identified as an acetal carbon (C-22), which is characteristic of spirostanol saponin. The ${ }^{13} \mathrm{C}$ signals of carbons near C-17, C-12 $\left(\delta_{\mathrm{C}} 32.8\right)$, $\mathrm{C}-13\left(\delta_{\mathrm{C}} 42.9\right), \mathrm{C}-14\left(\delta_{\mathrm{C}} 50.5\right), \mathrm{C}-16\left(\delta_{\mathrm{C}} 90.1\right)$, and C-21 $\left(\delta_{\mathrm{C}} 8.2\right)$ and the further long-range correlation from $21-\mathrm{CH}_{3}$ to the quaternary $\mathrm{C}$-atom at $\delta_{\mathrm{C}} 88.9(\mathrm{C}-17)$ was also observed, suggesting the presence of a hydroxyl group at C-17.

The above observations were supported by 2D-NMR analysis. The proton and protonated carbon resonances in the NMR spectrum of $\mathbf{1}$ were unambiguously assigned by the heteronuclear single quantum correlation (HSQC) experiment. Heteronuclear multiple bond correlations (HMBCs) of H-19/C-1, C-5, C-9, and C-10; H-4/C-2 and C-5; H-6/C-5, C-7, and C-8; H-8/C-9, C-11, and C-14; H-18/C-12, C-13, C-14, and C-17; H-15/H-14, H-16, and H-17; H-16/C-15, C-17, and C-22; $\mathrm{H}-21 / \mathrm{C}-17, \mathrm{C}-20$, and C-22; and $\mathrm{H}-27 / \mathrm{C}-24, \mathrm{C}-25$, and C-26 revealed the planar structure of 1 as spirostan-9(11)-en-3,17-diol (Figure 2). Meanwhile, in the nuclear Overhauser effect (NOE) spectrum, NOE correlations between $\mathrm{H} 19 / \mathrm{H}-4 \mathrm{~b}$ and $\mathrm{H}-8, \mathrm{H}-4 \mathrm{a} / \mathrm{H}-3$, and $\mathrm{H}-3 / \mathrm{H}-5$ indicated an $\alpha$-axial configuration of $\mathrm{H}-3$ and $\mathrm{H}-5$ and $\beta$ orientation of $\mathrm{H}-8, \mathrm{H}-19$, and $3-\mathrm{OH}$, with an $\mathrm{A} / \mathrm{B}$ trans ring junction pattern; whereas NOE correlations of $\mathrm{H}-19 / \mathrm{H}-8, \mathrm{H}-8 / \mathrm{H}-18, \mathrm{H}-18 / \mathrm{H}-15 \mathrm{~b}$, and H-20 and of H-15a/H-14 and $\mathrm{H}-16$ suggested $\alpha$-axial configurations for $\mathrm{H}-21$ and $17-\mathrm{OH}$ (D/E cis ring, Figure 3 ), $\beta$ orientation for $\mathrm{H}-18$, and B/C and C/D trans ring junction patterns. Finally, the absolute configuration of C-25 was deduced as $S$ based on the difference in chemical shifts between equatorial $\left(\delta_{\mathrm{H}} 3.24\right)$ and axial $\left(\delta_{\mathrm{H}} 4.03\right) \mathrm{H}-26$, which is usually $\Delta \delta>0.57$ and $<0.48 \mathrm{ppm}$ for $25 S$ and $25 R$ compounds, respectively [16]. Compound 1 was identified as (25S)-5 $\alpha$-spirostan-9(11)-en-3 $\beta, 17 \alpha$-diol (1; Figure 1). 


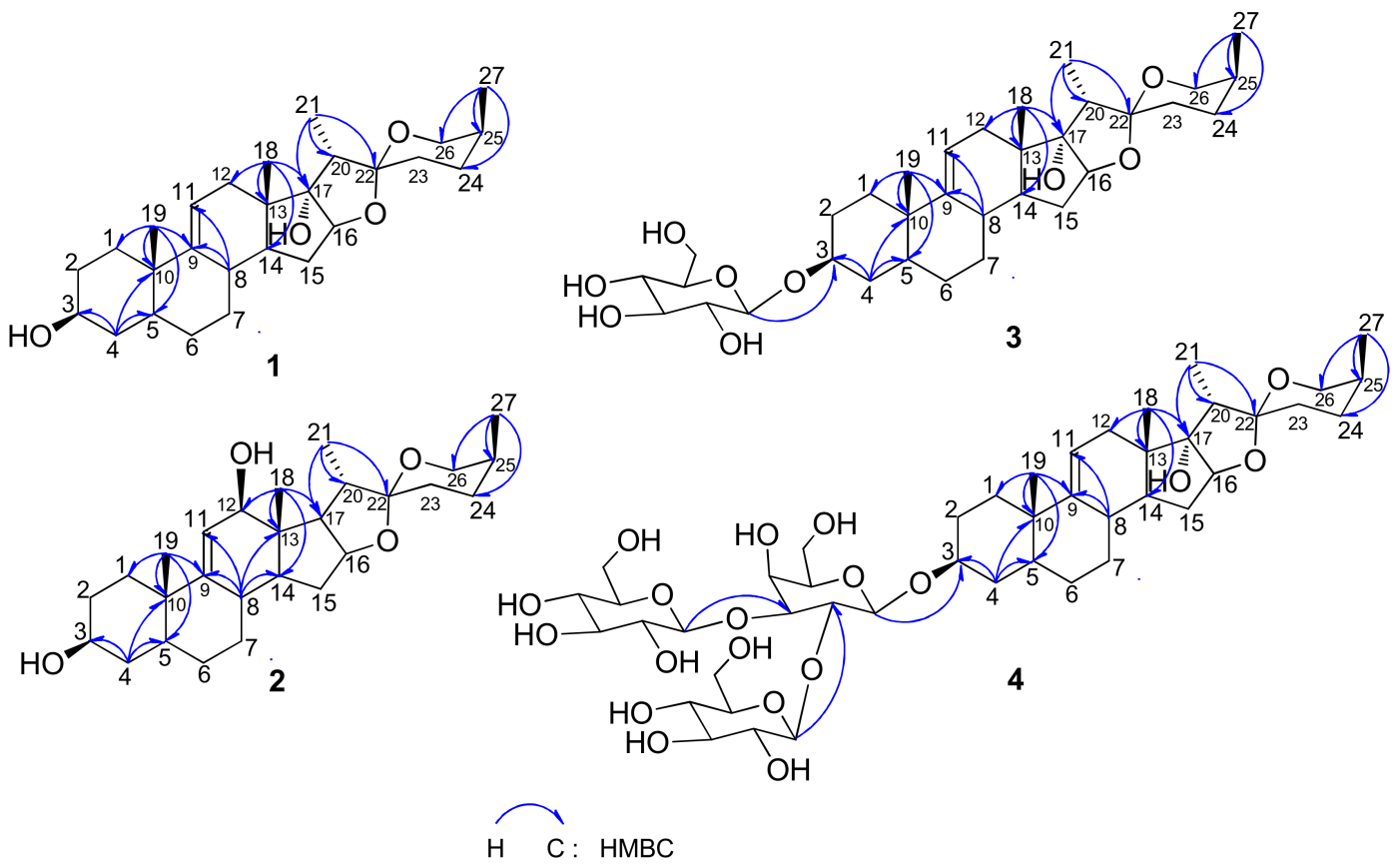

Figure 2. Key HMBC correlations of compounds 1-4.

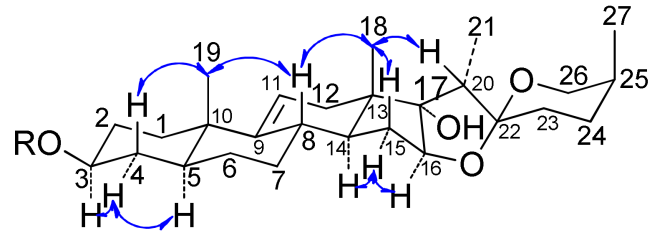

compounds 1, 3 and 4

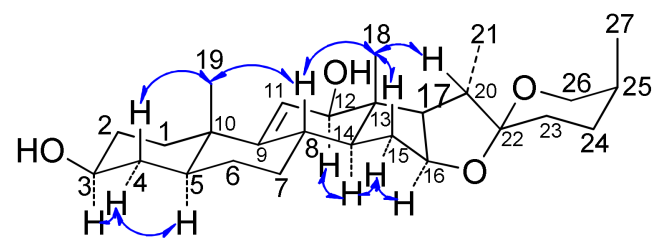

compound 2

$$
\overbrace{\mathrm{H}: \text { NOESY }}
$$

Figure 3. Key NOESY correlations of compounds 1-4.

Compound 2 was isolated as a white, amorphous powder and was positive to Liebermann-Burchard and Molisch chemical reactions. The HR-ESI-MS spectrum showed a positive molecular ion peak at $m / z 431.3152[\mathrm{M}+\mathrm{H}]^{+}$corresponding to a molecular formula of $\mathrm{C}_{27} \mathrm{H}_{42} \mathrm{O}_{4}$ (calculated for $431.3161[\mathrm{M}+\mathrm{H}]^{+}$). The ${ }^{1} \mathrm{H}-\mathrm{NMR}$ spectrum of $\mathbf{2}$ showed the presence of four methyl protons at $\delta_{\mathrm{H}} 1.09(3 \mathrm{H}, \mathrm{s}, \mathrm{H}-18), 0.99(3 \mathrm{H}, \mathrm{s}, \mathrm{H}-19), 1.42(3 \mathrm{H}, \mathrm{d}, J=6.9 \mathrm{~Hz}, \mathrm{H}-21)$, and $1.08(3 \mathrm{H}, \mathrm{d}$, $J=6.9 \mathrm{~Hz}, \mathrm{H}-27)$ and an exocyclic olefinic proton at $\delta_{\mathrm{H}} 5.59(1 \mathrm{H}$, broad singlet, $\mathrm{H}-11)$. The ${ }^{13} \mathrm{C}-\mathrm{NMR}$ spectrum showed 27 carbon signals-including one double-bond carbon at $\delta_{\mathrm{C}} 148.5$ (C-9) and 123.9 (C-11); four methyl groups at $\delta_{\mathrm{C}} 10.9$ (C-18), 18.0 (C-19), 14.0 (C-21), and 16.3 (C-27); and an quaternary carbon signal at $\delta_{\mathrm{C}} 110.1$ that was identified as an acetal carbon (C-22). A comparison of proton and carbon data showed that the resonances of $\mathbf{2}$ were similar to those of $\mathbf{1}$ (Table 1), except for the absence of $17-\mathrm{OH}$ and appearance of $12-\mathrm{OH}$ in the former. Moreover, the down-field shifted methylene signal at $\delta_{\mathrm{C}} 123.9$ (C-11) and the up-field Me-18 signal at $\delta_{\mathrm{C}} 10.9$ suggested the existence of one $\beta$-oriented hydroxyl group at C-12 $\left(\delta_{\mathrm{C}} 78.6\right)$, which was further confirmed by ROESY correlations between $\mathrm{H}-12$ and H-14, H-14 and H-15, H-15 and H-16. The chemical structure of 2 was supported by 2D-NMR data from HSQC, HMBC (Figure 2), and NOE spectroscopy (NOESY) (Figure 3) experiments. Compound 2 was identified as (25S)-5 $\alpha$-spirostan-9(11)-en-3 $\beta, 12 \beta$-diol (2; Figure 1). 
Table 1. ${ }^{1} \mathrm{H}-\mathrm{NMR}$ and ${ }^{13} \mathrm{C}-\mathrm{NMR}$ data for compounds $\mathbf{1 - 4}{ }^{\dagger}$.

\begin{tabular}{|c|c|c|c|c|c|c|c|c|}
\hline \multirow[b]{2}{*}{ No. } & \multicolumn{2}{|l|}{1} & \multicolumn{2}{|c|}{2} & \multicolumn{2}{|l|}{3} & \multicolumn{2}{|c|}{4} \\
\hline & $\delta \mathrm{H}$ & $\delta \mathrm{C}$ & $\delta \mathbf{H}$ & $\delta \mathrm{C}$ & $\delta \mathbf{H}$ & $\delta \mathrm{C}$ & $\delta H$ & $\delta \mathrm{C}$ \\
\hline 1 & $\begin{array}{l}1.44,1 \mathrm{H}, \text { са. } \\
1.75,1 \mathrm{H}, \text { са. }\end{array}$ & 35.3 & $\begin{array}{l}1.46,1 \mathrm{H}, \mathrm{ca} . \\
1.66,1 \mathrm{H}, \mathrm{ca} .\end{array}$ & 36.1 & $\begin{array}{l}1.26,1 \mathrm{H}, \text { ca. } \\
1.61,1 \mathrm{H}, \text { ca. }\end{array}$ & 35.3 & $\begin{array}{l}1.25,1 \mathrm{H}, \mathrm{ca} . \\
1.61,1 \mathrm{H}, \mathrm{ca} .\end{array}$ & 35.3 \\
\hline 2 & $\begin{array}{l}1.73,1 \mathrm{H}, \mathrm{ca} . \\
2.06,1 \mathrm{H}, \mathrm{ca} .\end{array}$ & 31.7 & $\begin{array}{l}1.67,1 \mathrm{H}, \mathrm{ca} . \\
2.19,1 \mathrm{H}, \mathrm{ca} .\end{array}$ & 32.9 & $\begin{array}{l}1.73,1 \mathrm{H}, \mathrm{ca} . \\
2.06,1 \mathrm{H}, \mathrm{ca} .\end{array}$ & 29.5 & $\begin{array}{l}1.73,1 \mathrm{H}, \mathrm{ca} . \\
2.06,1 \mathrm{H}, \mathrm{ca} .\end{array}$ & 29.5 \\
\hline 3 & $3.84,1 \mathrm{H}, \mathrm{ca}$ & 69.6 & $3.86,1 \mathrm{H}, \mathrm{ca}$. & 70.4 & $3.97,1 \mathrm{H}, \mathrm{ca}$. & 76.4 & $3.94,1 \mathrm{H}, \mathrm{ca}$. & 76.7 \\
\hline 4 & $\begin{array}{l}1.51,1 \mathrm{H}, \mathrm{ca} . \\
1.76,1 \mathrm{H}, \mathrm{ca} .\end{array}$ & 34.4 & $\begin{array}{l}1.53,1 \mathrm{H}, \mathrm{ca} . \\
1.84,1 \mathrm{H}, \mathrm{ca} .\end{array}$ & 39.3 & $\begin{array}{l}1.36,1 \mathrm{H}, \text { ca. } \\
1.87,1 \mathrm{H}, \text { ca. }\end{array}$ & 34.4 & $\begin{array}{l}1.36,1 \mathrm{H}, \mathrm{ca} . \\
1.88,1 \mathrm{H}, \mathrm{ca} .\end{array}$ & 34.4 \\
\hline 5 & $1.25,1 \mathrm{H}, \mathrm{ca}$ & 42.9 & $1.23,1 \mathrm{H}, \mathrm{ca}$. & 43.7 & $1.03,1 \mathrm{H}, \mathrm{ca}$ & 42.6 & $1.06,1 \mathrm{H}, \mathrm{ca}$. & 42.7 \\
\hline 6 & $\begin{array}{l}1.22,1 \mathrm{H}, \text { ca. } \\
1.38,1 \mathrm{H}, \text { ca. }\end{array}$ & 28.1 & $\begin{array}{l}1.24,1 \mathrm{H}, \mathrm{ca} \\
1.28,1 \mathrm{H}, \mathrm{ca}\end{array}$ & 28.9 & $\begin{array}{l}1.16,1 \mathrm{H}, \text { ca. } \\
1.25,1 \mathrm{H}, \text { ca. }\end{array}$ & 28.2 & $\begin{array}{l}1.18,1 \mathrm{H}, \mathrm{ca} . \\
1.24,1 \mathrm{H}, \mathrm{ca} .\end{array}$ & 28.2 \\
\hline 7 & $\begin{array}{l}0.95,1 \mathrm{H}, \text { ca. } \\
1.75,1 \mathrm{H}, \text { ca. }\end{array}$ & 33 & $\begin{array}{l}0.94,1 \mathrm{H}, \mathrm{ca} . \\
1.81,1 \mathrm{H}, \mathrm{ca} .\end{array}$ & 33.5 & $\begin{array}{l}0.92,1 \mathrm{H}, \mathrm{ca} . \\
1.73,1 \mathrm{H}, \mathrm{ca} .\end{array}$ & 33 & $\begin{array}{l}0.93,1 \mathrm{H}, \mathrm{ca} . \\
1.73,1 \mathrm{H}, \mathrm{cc} .\end{array}$ & 33 \\
\hline 8 & $2.24,1 \mathrm{H}, \mathrm{ca}$. & 36.1 & $2.18,1 \mathrm{H}, \mathrm{ca}$. & 36.3 & $2.09,1 \mathrm{H}$, ca. & 36.2 & $2.08,1 \mathrm{H}, \mathrm{ca}$. & 36.2 \\
\hline 9 & - & 145.5 & - & 148.5 & - & 145.5 & - & 145.5 \\
\hline 10 & - & 37.5 & - & 38.2 & - & 37.6 & - & 37.6 \\
\hline 11 & $\begin{array}{l}5.51,1 \mathrm{H}, \mathrm{d} \\
J=5.3 \mathrm{~Hz}\end{array}$ & 116.2 & $5.59,1 \mathrm{H}, \mathrm{brs}$ & 123.9 & $\begin{array}{c}5.45,1 \mathrm{H}, \mathrm{d} \\
J=5.3 \mathrm{~Hz}\end{array}$ & 116.4 & $\begin{array}{c}5.45,1 \mathrm{H}, \mathrm{d} \\
J=5.3 \mathrm{~Hz}\end{array}$ & 116.4 \\
\hline 12 & $\begin{array}{l}1.78,1 \mathrm{H}, \mathrm{ca} . \\
3.07,1 \mathrm{H}, \mathrm{d} \\
I=17.4 \mathrm{~Hz}\end{array}$ & 32.8 & $4.33,1 \mathrm{H}, \mathrm{brs}$ & 78.6 & $\begin{array}{l}1.77,1 \mathrm{H}, \mathrm{ca} . \\
3.06,1 \mathrm{H}, \mathrm{d} \\
J=17.4 \mathrm{~Hz}\end{array}$ & 33 & $\begin{array}{l}1.78,1 \mathrm{H}, \mathrm{ca} . \\
3.06,1 \mathrm{H}, \mathrm{d} \\
I=17.4 \mathrm{~Hz}\end{array}$ & 33 \\
\hline 13 & - & 42.9 & - & 45.2 & - & 43.1 & - & 43.1 \\
\hline 14 & $2.13,1 \mathrm{H}, \mathrm{ca}$. & 50.5 & $1.48,1 \mathrm{H}, \mathrm{ca}$. & 53.2 & $2.13,1 \mathrm{H}, \mathrm{ca}$ & 50.6 & $2.13,1 \mathrm{H}, \mathrm{ca}$. & 50.6 \\
\hline 15 & $\begin{array}{l}1.51,1 \mathrm{H}, \mathrm{ca} . \\
2.32,1 \mathrm{H}, \mathrm{ca} .\end{array}$ & 31.9 & $\begin{array}{l}1.52,1 \mathrm{H}, \mathrm{ca} . \\
2.07,1 \mathrm{H}, \mathrm{ca} .\end{array}$ & 32.4 & $\begin{array}{l}1.53,1 \mathrm{H}, \mathrm{ca} . \\
2.32,1 \mathrm{H}, \mathrm{ca} .\end{array}$ & 32.1 & $\begin{array}{l}1.52,1 \mathrm{H}, \mathrm{ca} . \\
2.31,1 \mathrm{H}, \mathrm{ca} .\end{array}$ & 32.1 \\
\hline 16 & $\begin{array}{l}4.45,1 \mathrm{H}, \mathrm{d} \\
J=7.2 \mathrm{~Hz}\end{array}$ & 90.1 & $4.62,1 \mathrm{H}, \mathrm{ca}$. & 81.5 & $\begin{array}{l}4.48,1 \mathrm{H}, \mathrm{d} \\
J=7.2 \mathrm{~Hz}\end{array}$ & 90.3 & $\begin{array}{l}4.45,1 \mathrm{H}, \mathrm{d} \\
J=7.2 \mathrm{~Hz}\end{array}$ & 90.3 \\
\hline 17 & - & 88.9 & $\begin{array}{l}2.34,1 \mathrm{H}, \mathrm{t} \\
J=6.0 \mathrm{~Hz}\end{array}$ & 61.9 & - & 89.1 & - & 89.1 \\
\hline 18 & $0.92,3 \mathrm{H}, \mathrm{s}$ & 16.4 & $0.1 .09,3 \mathrm{H}, \mathrm{s}$ & 10.9 & $0.92,3 \mathrm{H}, \mathrm{s}$ & 16.6 & $0.92,3 \mathrm{H}, \mathrm{s}$ & 16.6 \\
\hline 19 & $0.98,3 \mathrm{H}, \mathrm{s}$ & 17.4 & $0.99,3 \mathrm{H}, \mathrm{s}$ & 18.0 & $0.68,3 \mathrm{H}, \mathrm{s}$ & 17.4 & $0.84,3 \mathrm{H}, \mathrm{s}$ & 17.4 \\
\hline 20 & $2.23,1 \mathrm{H}, \mathrm{ca}$. & 44.8 & $2.14,1 \mathrm{H}, \mathrm{ca}$ & 43.8 & $2.21,1 \mathrm{H}, \mathrm{ca}$ & 45.1 & $2.22,1 \mathrm{H}, \mathrm{ca}$ & 45.1 \\
\hline 21 & $\begin{array}{l}1.21,3 \mathrm{H}, \mathrm{d} \\
J=7.1 \mathrm{~Hz}\end{array}$ & 8.2 & $\begin{array}{l}1.42,3 \mathrm{H}, \mathrm{d} \\
J=6.9 \mathrm{~Hz}\end{array}$ & 14.0 & $\begin{array}{l}1.23,3 \mathrm{H}, \mathrm{d} \\
J=7.1 \mathrm{~Hz}\end{array}$ & 8.5 & $\begin{array}{l}1.22,3 \mathrm{H}, \mathrm{d} \\
J=7.1 \mathrm{~Hz}\end{array}$ & 8.5 \\
\hline 22 & - & 110.5 & - & 110.1 & - & 109.8 & - & 109.8 \\
\hline 23 & $\begin{array}{l}1.45,1 \mathrm{H}, \mathrm{ca} . \\
1.92,1 \mathrm{H}, \mathrm{ca} .\end{array}$ & 25.6 & $\begin{array}{l}1.47,1 \mathrm{H}, \mathrm{ca} . \\
1.92,1 \mathrm{H}, \mathrm{ca}\end{array}$ & 26.4 & $\begin{array}{l}1.46,1 \mathrm{H}, \mathrm{ca} . \\
1.92,1 \mathrm{H}, \mathrm{ca} .\end{array}$ & 25.8 & $\begin{array}{l}1.46,1 \mathrm{H}, \mathrm{ca} . \\
1.91,1 \mathrm{H}, \mathrm{ca} .\end{array}$ & 25.8 \\
\hline 24 & $\begin{array}{l}1.32,1 \mathrm{H}, \mathrm{ca} . \\
2.06,1 \mathrm{H}, \mathrm{ca}\end{array}$ & 24.8 & $\begin{array}{l}1.34,1 \mathrm{H}, \mathrm{ca} . \\
2.04,1 \mathrm{H}, \mathrm{ca}\end{array}$ & 26.2 & $\begin{array}{l}1.33,1 \mathrm{H}, \text { ca. } \\
2.05,1 \mathrm{H}, \mathrm{ca} .\end{array}$ & 25.1 & $\begin{array}{l}1.32,1 \mathrm{H}, \mathrm{ca} . \\
2.03,1 \mathrm{H}, \mathrm{ca} .\end{array}$ & 25.1 \\
\hline 25 & $\begin{array}{l}1.55,1 \mathrm{H}, \mathrm{ca} . \\
3.24,1 \mathrm{H}, \mathrm{d} \\
J=11.0 \mathrm{~Hz}\end{array}$ & 26.5 & $\begin{array}{l}1.59,1 \mathrm{H}, \mathrm{ca} . \\
3.33,1 \mathrm{H}, \mathrm{d} \\
J=11.0 \mathrm{~Hz}\end{array}$ & 27.6 & $\begin{array}{l}1.55,1 \mathrm{H}, \mathrm{ca} . \\
3.24,1 \mathrm{H}, \mathrm{d} \\
J=11.0 \mathrm{~Hz}\end{array}$ & 26.3 & $\begin{array}{l}1.54,1 \mathrm{H}, \mathrm{ca} . \\
3.24,1 \mathrm{H}, \mathrm{d} \\
J=11.0 \mathrm{~Hz}\end{array}$ & 26.7 \\
\hline & $\begin{array}{c}4.04,1 \mathrm{H}, \mathrm{dd} \\
J=2.4,11.0 \mathrm{~Hz}\end{array}$ & & $\begin{array}{c}4.09,1 \mathrm{H}, \mathrm{dd}, \\
J=2.4,11.0 \\
\mathrm{~Hz}\end{array}$ & & $\begin{array}{c}4.03,1 \mathrm{H}, \mathrm{dd} \\
J=2.4,11.0 \mathrm{~Hz}\end{array}$ & & $\begin{array}{c}4.04,1 \mathrm{H}, \mathrm{dd} \\
J=2.4,11.0 \mathrm{~Hz}\end{array}$ & \\
\hline 27 & $\begin{array}{c}1.05,3 \mathrm{H}, \mathrm{d} \\
J=7.1 \mathrm{~Hz}\end{array}$ & 15.4 & $\begin{array}{l}1.08,3 \mathrm{H}, \mathrm{d} \\
J=6.9 \mathrm{~Hz}\end{array}$ & 16.3 & $\begin{array}{c}1.05,3 \mathrm{H}, \mathrm{d} \\
J=7.1 \mathrm{~Hz}\end{array}$ & 15.6 & $\begin{array}{c}1.06,3 \mathrm{H}, \mathrm{d} \\
J=7.1 \mathrm{~Hz}\end{array}$ & 15.6 \\
\hline Sugar & & & & & & & & \\
\hline Gal-1 & & & & & $\begin{array}{l}4.98,1 \mathrm{H}, \mathrm{d} \\
J=7.6 \mathrm{~Hz}\end{array}$ & 102.2 & $\begin{array}{l}4.91,1 \mathrm{H}, \mathrm{d} \\
J=7.6 \mathrm{~Hz}\end{array}$ & 101.8 \\
\hline 2 & & & & & $4.47,1 \mathrm{H}$, ca. & 72.1 & $4.62,1 \mathrm{H}, \mathrm{ca}$ & 80.5 \\
\hline 3 & & & & & $\begin{array}{l}4.18,1 \mathrm{H}, \mathrm{t} \\
J=6.0 \mathrm{~Hz}\end{array}$ & 76.4 & $4.18,1 \mathrm{H}, \mathrm{ca}$. & 85.5 \\
\hline 4 & & & & & $\begin{array}{l}4.61,1 \mathrm{H}, \mathrm{d} \\
J=3.0 \mathrm{~Hz}\end{array}$ & 69.8 & $4.53,1 \mathrm{H}, \mathrm{ca}$ & 72.7 \\
\hline 5 & & & & & $\begin{array}{r}4.24,1 \mathrm{H}, \mathrm{dd}, \\
J=3.0,9.4 \mathrm{~Hz}\end{array}$ & 74.9 & $4.01,1 \mathrm{H}, \mathrm{ca}$. & 77.6 \\
\hline 6 & & & & & $4.48,2 \mathrm{H}, \mathrm{ca}$ & 62 & $\begin{array}{l}4.26,1 \mathrm{H}, \mathrm{ca} . \\
4.80,1 \mathrm{H}, \mathrm{ca} .\end{array}$ & 59.9 \\
\hline Glc-1' & & & & & & & $\begin{array}{l}5.16,1 \mathrm{H}, \mathrm{d} \\
J=7.5 \mathrm{~Hz}\end{array}$ & 104.6 \\
\hline $2^{\prime}$ & & & & & & & $4.13,1 \mathrm{H}, \mathrm{ca}$. & 75 \\
\hline $3^{\prime}$ & & & & & & & $3.81,1 \mathrm{H}, \mathrm{ca}$. & 78.3 \\
\hline $4^{\prime}$ & & & & & & & $3.99,1 \mathrm{H}, \mathrm{ca}$. & 71.2 \\
\hline $5^{\prime}$ & & & & & & & $4.07,1 \mathrm{H}, \mathrm{ca}$. & 76.2 \\
\hline $6^{\prime}$ & & & & & & & $\begin{array}{l}4.02,1 \mathrm{H}, \mathrm{ca} . \\
4.65,1 \mathrm{H}, \mathrm{ca}\end{array}$ & 60.9 \\
\hline Glc- $1^{\prime \prime}$ & & & & & & & $\begin{array}{l}5.27,1 \mathrm{H}, \mathrm{d} \\
J=7.5 \mathrm{~Hz}\end{array}$ & 106.4 \\
\hline $2^{\prime \prime}$ & & & & & & & $4.06,1 \mathrm{H}, \mathrm{ca}$. & 74.5 \\
\hline $3^{\prime \prime}$ & & & & & & & $3.97,1 \mathrm{H}, \mathrm{ca}$. & 77.9 \\
\hline $4^{\prime \prime}$ & & & & & & & $4.26,1 \mathrm{H}, \mathrm{ca}$. & 69.6 \\
\hline $5^{\prime \prime}$ & & & & & & & $4.12,1 \mathrm{H}, \mathrm{ca}$. & 77 \\
\hline $6^{\prime \prime}$ & & & & & & & $\begin{array}{l}4.37,1 \mathrm{H}, \mathrm{ca} . \\
4.59,1 \mathrm{H}, \mathrm{ca} .\end{array}$ & 62.6 \\
\hline
\end{tabular}

${ }^{\dagger}$ Assignments aided by the HSQC, HMBC, and NOESY experiments; ${ }^{1} \mathrm{H}$ - and ${ }^{13} \mathrm{C}-\mathrm{NMR}$ were measured at 400 and $100 \mathrm{MHz}$ in pyridine- $d_{5}$. 
Compound 3 was isolated as a white, amorphous powder and was positive to Liebermann-Burchard and Molisch chemical reactions. The HR-ESI-MS spectrum showed a negative molecular ion peak at $m / z 591.3508[\mathrm{M}+\mathrm{H}]^{-}$corresponding to a molecular formula of $\mathrm{C}_{33} \mathrm{H}_{52} \mathrm{O}_{9}$ (calculated for $591.3533[\mathrm{M}+\mathrm{H}]^{-}$). The ${ }^{1} \mathrm{H}-\mathrm{NMR}$ spectrum of 3 revealed the presence of four methyl protons at $\delta_{\mathrm{H}} 0.92(3 \mathrm{H}, \mathrm{s}, \mathrm{H}-18), 0.68(3 \mathrm{H}, \mathrm{s}, \mathrm{H}-19), 1.22(3 \mathrm{H}, \mathrm{d}, J=7.1 \mathrm{~Hz}, \mathrm{H}-21)$, and $1.05(3 \mathrm{H}, \mathrm{d}$, $J=7.1 \mathrm{~Hz}, \mathrm{H}-27)$ and an anomeric proton at $\delta_{\mathrm{H}} 4.98(1 \mathrm{H}, \mathrm{d}, J=7.6 \mathrm{~Hz}, \mathrm{H}-\mathrm{Glc}-1)$. The ${ }^{13} \mathrm{C}-\mathrm{NMR}$ spectrum showed 33 carbon signals; one double-bond carbon at $\delta_{\mathrm{C}} 145.5$ (C-9) and 116.4 (C-11) and four methyl groups at $\delta_{\mathrm{C}} 16.6(\mathrm{C}-18), 17.4(\mathrm{C}-19), 8.5(\mathrm{C}-21)$, and $15.6(\mathrm{C}-27)$, as well as an anomeric carbon at $\delta_{\mathrm{C}}$ 102.2 (C-Glc-1). In addition, the quaternary carbon signal at $\delta_{\mathrm{C}} 109.8$ was identified as an acetal carbon (C-22), which is characteristic of spirostanol saponin. A comparison of the proton and carbon data with those of compound 1 (Table 1 ) indicated that a glucose unit $(102.2,72.1,76.4,69.8,74.9$, and 62.0) was linked to the $\mathrm{C}-3$ of the aglycone $\left(\mathrm{C}_{3}+6.8 \mathrm{ppm}\right)$. Acid hydrolysis of 3 yielded D-glucose, which was confirmed by GC analysis of the trimethylsilyl-l-cysteine derivatives of compound 3 hydrolysate and authentic sugars. The large $J$ values $(J>7.0 \mathrm{~Hz})$ of the anomeric proton signals reflected the $\beta$ configuration of D-glucose. Compound 3 was identified as (25S)- $5 \alpha$-spirostan-9(11)-en-3 $\beta, 17 \alpha$-diol 3-O- $\beta$-D-glucopyranoside (3; Figure 1) based on HSQC, HMBC (Figure 2), and NOESY (Figure 3) data.

Compound 4 was isolated as a white, amorphous powder and was positive to Liebermann-Burchard and Molisch chemical reactions. The HR-ESI-MS spectrum showed a negative molecular ion peak at $m / z 915.4565\left[\mathrm{M}+\mathrm{H}^{-}\right.$, corresponding to a molecular formula of $\mathrm{C}_{45} \mathrm{H}_{72} \mathrm{O}_{19}$ (calculated for $915.4590\left[\mathrm{M}+\mathrm{H}^{-}\right.$). NMR spectra for 4 and 1 showed similar features, except that the $3-\mathrm{OH}$ in 1 was replaced by $\beta$-D-glucopyranosyl-( $1 \rightarrow 2)$ [ $\beta$-D-glucopyranosyl-( $1 \rightarrow 3)$ ]- $\beta$-D-galactopyranoside in 4 . The HMBC spectrum of 4 (Figure 2) revealed correlations between $\mathrm{H}-\mathrm{Glc}-1^{\prime \prime} / \mathrm{C}-\mathrm{Gal}-3$, H-Glc-1'/C-Gal-2, and H-Gal-1/C-3, with the two glucose units linked to C-Gal-2 and C-Gal-3 of the inner galactose unit, which was itself linked to C-3 of aglycone. This was confirmed by acid hydrolysis and subsequent GC analysis of the hydrolysates and 2D-NMR analysis. Coupling constants of the anomeric proton signals suggested $\beta$-configuration of D-glucose and D-galactose. Based on this evidence, compound 4 was identified as (25S)- $5 \alpha$-spirostan-9(11)-en-3 $\beta, 17 \alpha$-diol 3-O- $\beta$-D-glucopyranosyl- $(1 \rightarrow 2)$ [ $\beta$-D-glucopyranosyl- $(1 \rightarrow 3)]$ - $\beta$-D-galactopyranoside ( 4 ; Figure 1$)$.

The two known glycosides were identified as japonicoside $\mathrm{B}(5$, Figure 1$)$ and japonicoside C (6, Figure 1); (25S)- $5 \alpha$-spirostan-9(11)-en-3 $\beta, 17 \alpha$-diol 3-O- $\beta$-D-glucopyranosyl- $(1 \rightarrow 2)$ [ $\beta$-D-xylopyranosyl-( $(\rightarrow 3)]$ - $\beta$-D-glucopyranosyl $(1 \rightarrow 4)-\beta$-D-galactopyranoside $(5), \quad(25 S)$ - $5 \alpha$-spirostan9(11)-en-3 $\beta, 17 \alpha, 24 \alpha$-diol 3-O- $\beta$-D-glucopyranosyl-( $1 \rightarrow 2)$-[ $\beta$-D-xylopyranosyl-(1 $\rightarrow 3)]-\beta$-D-glucopyranosyl $(1 \rightarrow 4)-\beta$-D-galactopyranoside (6), respectively, by comparing their spectroscopic data (Supplementary Materials) with those reported in the literature [6].

Because steroidal saponins have been reported to possess varying cytotoxic activity against various cancer cell lines [17-19], in this paper, the cytotoxic activity against human SMMC-7712, Bel-7402, A549, H460, and K562 tumor cells of compounds 1-6 were evaluated by MTT method and all the compounds exhibited cytotoxicity with the cell lines. Compound 2 exhibited a more potent antitumor effect than 1: it seemed that the presence of a free hydroxyl group at C-12 was more potent than a free hydroxyl group at C-17. Compounds 1, 3-5 shared the same aglycone, but exhibited different activities. This suggested that the structural differences such as the category, the number, and the sequence of the oligosaccharide chain at C-3 played a role in terms of antitumor effect. Meanwhile, compared with 5 , compound $\mathbf{6}$ has one more free hydroxyl group than 5, but their cytotoxic activity have no differences. It seemed that the presence of a free hydroxyl group at C-24 has less effect on the cytotoxic activity. Furthermore, compounds 2 and 4 exhibited moderate cytotoxicity against A549 cells with $\mathrm{IC}_{50}$ values of $14.4 \mu \mathrm{M}$ and $12.3 \mu \mathrm{M}$, respectively, while compounds $\mathbf{1}, \mathbf{3}, \mathbf{5}$ and $\mathbf{6}$ displayed no activity $\left(\mathrm{IC}_{50}>100 \mu \mathrm{M}\right)$. It seemed that the differences of the composition of the oligosaccharide chain have an effect on the cytotoxic activity and selectivity of the various cell lines. Thus, our results indicated that the antitumor effects of steroidal constituents from this species are very sensitive to their 
precise functionalization. Therefore, more extensive studies are needed before a clear structure-activity relationship can be reached.

\section{Materials and Methods}

\subsection{Materials}

Optical rotations were recorded in $\mathrm{MeOH}$ using a Rudolph research analytical automatic polarimeter (Rudolph Research Analytical, Hackettstown, NJ, USA). Infrared (IR) spectra were recorded on a TENSOR-27 instrument (Bruker, Billerica, MA, USA). High-resolution electrospray ionization mass spectrometry (HR-ESI-MS) analysis was carried out on a model 6550 quadrupole time-of-flight mass spectrometer (Agilent Technologies, Santa Clara, CA, USA). One-dimensional (1D) and 2D nuclear magnetic resonance (NMR) spectra were recorded on an AVANCE400 instrument (Bruker) with tetramethylsilane as an internal standard. High-performance liquid chromatography (HPLC) was performed on a 2695 Separations Module (Waters, Milford, MA, USA) coupled with a 2996 Photodiode Array Detector and octadecylsilyl (ODS)-3 column $(4.6 \times 250 \mathrm{~mm}$, $5 \mathrm{~mm}$ particles; COSMOSIL, Tokyo, Japan). Semi-preparative HPLC was performed on a LC-6AD pump equipped with a SPD-20A ultraviolet detector and an Ultimate XB-C18 column $(10 \times 250 \mathrm{~mm}$, $5 \mathrm{~mm}$ particles) (Shimadzu, Kyoto, Japan). Gas chromatography (GC) was performed on an $7890 \mathrm{~A}$ chromatograph (Agilent Technologies, Santa Clara, CA, USA) equipped with an HP-5 capillary column ( $30 \mathrm{~m} \times 320 \mathrm{~mm} \times 0.25 \mathrm{~mm}$; Agilent Technologies). Sephadex LH-20 and C-18 (40-75 mm) silica gels were purchased from GE Healthcare Bio-Sciences AB (Uppsala, Sweden), and silica gel was also purchased from Qingdao Haiyang Chemical Group Corporation (Qingdao, China).

\subsection{Plant Material}

Smilacina japonica A. Gray was collected in August of 2014 from the Taibai region of the Qinba Mountains in the Shaanxi Province of China and was authenticated by Professor Jitao Wang (Shaanxi University of Chinese Medicine). A voucher specimen (herbarium no. 20140826) has been deposited in the Medicinal Plants Herbarium, Shaanxi University of Chinese Medicine (Xianyang, China).

\subsection{Extraction and Isolation}

The air-dried powder of S. japonica rhizomes and roots of $(6.3 \mathrm{~kg})$ was extracted three times with $80 \% \mathrm{EtOH}$ under reflux at $80{ }^{\circ} \mathrm{C}$. After removing the solvent, the concentrated residue was successively partitioned with petroleum ether and $n-\mathrm{BuOH}$. The $n-\mathrm{BuOH}$ extract $(200 \mathrm{~g})$ was subjected to column chromatography $(\mathrm{CC})$ on silica gel with gradient elution $\left(\mathrm{CHCl}_{3}-\mathrm{MeOH}-\mathrm{H}_{2} \mathrm{O}, 100: 0: 0-65: 35: 10\right)$, which yielded five fractions (Fr.1-5). Fr.1 (10.5 g) was subjected to CC on silica gel; elution with $\mathrm{CHCl}_{3}-\mathrm{MeOH}-\mathrm{H}_{2} \mathrm{O}$ (100:0:0-10:1:0.1) yielded compound 1 (8.3 mg) and compound 2 (6.8 mg). Fr.4 (22 g) was subjected to CC on silica gel; elution with $\mathrm{CHCl}_{3}-\mathrm{MeOH}-\mathrm{H}_{2} \mathrm{O}$ (100:0:0-70:30:5) yielded four subfractions (Fr.4-1-4-4). Fr.4-1 (1.6 g) was subjected to CC on ODS gel; elution with $\mathrm{MeOH}-\mathrm{H}_{2} \mathrm{O}$ (10:90-70:30) yielded compound 3 (15.5 mg). Fr.4-3 (3.4 g) was subjected to CC on ODS gel; elution with $\mathrm{MeOH}-\mathrm{H}_{2} \mathrm{O}$ (10:90-70:30) yielded three subfractions (Fr.4-3-1-4-3-3). Fr.4-3-2 (188 mg) was purified by HPLC (flow rate: $1.5 \mathrm{~mL} / \mathrm{min}$ ) with $\mathrm{MeCN}-\mathrm{H}_{2} \mathrm{O}$ (46:54) as the mobile phase, yielding compound 4 (18.6 mg; retention time $\left.\left[t_{R}\right]=41.2 \mathrm{~min}\right)$, compound $5\left(37.6 \mathrm{mg} ; t_{\mathrm{R}}=33.4 \mathrm{~min}\right)$, and compound 6 $\left(11.6 \mathrm{mg} ; t_{\mathrm{R}}=31.2 \mathrm{~min}\right)$.

\section{4. (25S)-5 $\alpha$-Spirostan-9(11)-en-3 $\beta, 17 \alpha$-diol (Compound 1)}

This compound was a white amorphous powder (purity $>98 \%$ ) with the following spectral features. $[\alpha]_{\mathrm{D}}^{23.1}-31.5$ ( c 1.57, MeOH); IR (KBr) $v_{\max }$ : 3407, 2934, 1653, 1072, 1035, 979, 913, 892, $845 \mathrm{~cm}^{-1} ;{ }^{1} \mathrm{H}$ - and ${ }^{13} \mathrm{C}-\mathrm{NMR}$ spectral data (Table 1): positive HR-ESI-MS $\mathrm{m} / z 431.3154[\mathrm{M}+\mathrm{H}]^{+}$ (calculated for $\mathrm{C}_{27} \mathrm{H}_{43} \mathrm{O}_{4}: 431.3161[\mathrm{M}+\mathrm{H}]^{+}$). 


\section{5. (25S)-5 $\alpha$-Spirostan-9(11)-en-3 $\beta, 12 \beta$-diol (Compound 2)}

This compound was a white amorphous powder (purity of 94\%) with the following spectral features. $[\alpha]_{\mathrm{D}}^{23.3}-39.0$ (c 0.61, MeOH); IR (KBr) $v_{\max }: 3407,2929,1664,1070,1037,979,915,893$, $847 \mathrm{~cm}^{-1} ;{ }^{1} \mathrm{H}$ - and ${ }^{13} \mathrm{C}-\mathrm{NMR}$ spectral data (Table 1): positive HR-ESI-MS $\mathrm{m} / z 431.3152[\mathrm{M}+\mathrm{H}]^{+}$ (calculated for $\mathrm{C}_{27} \mathrm{H}_{43} \mathrm{O}_{4}: 431.3161[\mathrm{M}+\mathrm{H}]^{+}$).

\section{6. (25S)-5 $\alpha$-Spirostan-9(11)-en-3 $\beta, 17 \alpha$-diol 3-O- $\beta$-D-glucopyranoside (Compound 3)}

This compound was a white amorphous powder (purity $>98 \%$ ) with the following spectral features. $[\alpha]_{\mathrm{D}}^{22.9}-96.6(c 0.16, \mathrm{MeOH})$; IR (KBr) $v_{\max }: 3410,2939,1658,1071,1037,978,916,893$, $845 \mathrm{~cm}^{-1} ;{ }^{1} \mathrm{H}$ - and ${ }^{13} \mathrm{C}-\mathrm{NMR}$ spectral data (Table 1 ): negative HR-ESI-MS $\mathrm{m} / z$ 591.3508 [M - H] ${ }^{-}$ (calculated for $\mathrm{C}_{33} \mathrm{H}_{51} \mathrm{O}_{9}: 591.3533[\mathrm{M}-\mathrm{H}]^{-}$).

3.7. (25S)-5 $\alpha$-Spirostan-9(11)-en-3 $\beta, 17 \alpha$-diol3-O- $\beta$-D-glucopyranosyl-(1 $\rightarrow 2)$-[ $\beta$-D-glucopyranosyl- $(1 \rightarrow 3)]-$ $\beta$-D-galactopyranoside (Compound 4)

This compound was a white amorphous powder (purity $>98 \%$ ) with the following spectral features. $[\alpha]_{\mathrm{D}}^{23.0}-21.3\left(c\right.$ 0.70, MeOH); IR (KBr) $v_{\max }: 3410,2930,1652,1072,1035,977,915,893$, $847 \mathrm{~cm}^{-1} ;{ }^{1} \mathrm{H}$ - and ${ }^{13} \mathrm{C}-\mathrm{NMR}$ spectral data (Table 1): negative HR-ESI-MS $\mathrm{m} / z$ 915.4565 [M - H] ${ }^{-}$ (calculated for $\mathrm{C}_{45} \mathrm{H}_{71} \mathrm{O}_{19}: 915.4590[\mathrm{M}-\mathrm{H}]^{-}$).

\subsection{Acid Hydrolysis of Compounds 3-4 and Determination of Absolute Configuration of Sugars}

Compounds 3 and 4 ( 3 and $4 \mathrm{mg}$ ) were individually hydrolyzed with $1 \mathrm{~N} \mathrm{HCl}$-dioxane (1:1, $3 \mathrm{~mL})$ at $60{ }^{\circ} \mathrm{C}$ for $6 \mathrm{~h}$. After dilution with $\mathrm{H}_{2} \mathrm{O}(5 \mathrm{~mL})$, the reaction mixture was extracted with EtOAc, yielding distinct EtOAc and $\mathrm{H}_{2} \mathrm{O}$ phases. The latter was evaporated under reduced pressure. After adding $\mathrm{H}_{2} \mathrm{O}(5 \mathrm{~mL})$, the acidic solution was evaporated again; this procedure was repeated until a neutral solution was obtained. This was then evaporated and dried in a vacuum, yielding a monosaccharide residue that was dissolved in pyridine $(0.5 \mathrm{~mL}) ; 2 \mathrm{mg}$ of 1-cysteine methyl ester hydrochloride was added and the mixture was maintained at $60{ }^{\circ} \mathrm{C}$ for $2 \mathrm{~h}$, evaporated under a stream of $\mathrm{N}_{2}$, and dried in a vacuum. After adding $0.2 \mathrm{~mL}$ of $\mathrm{N}$-trimethylsilylimidazole, the mixture was maintained at $60{ }^{\circ} \mathrm{C}$ for $1 \mathrm{~h}$ and then partitioned between n-hexane and $\mathrm{H}_{2} \mathrm{O}(2 \mathrm{~mL}$ each). The n-hexane extract was analyzed by GC under the following conditions: HP-5 capillary column $(30 \mathrm{~m} \times 320 \mathrm{~mm} \times 0.25 \mu \mathrm{m})$; flame ionization detector; detector temperature $=280{ }^{\circ} \mathrm{C}$; injection temperature $=250{ }^{\circ} \mathrm{C}$; initial temperature $=100{ }^{\circ} \mathrm{C}$ for $2 \mathrm{~min}$, followed by an increase to $280{ }^{\circ} \mathrm{C}$ at a rate of $10{ }^{\circ} \mathrm{C} / \mathrm{min}$; final temperature $=280{ }^{\circ} \mathrm{C}$ for $5 \mathrm{~min}$; and $\mathrm{N}_{2}$ gas as a carrier. The absolute configurations of sugars isolated from the hydrolysates of compounds $\mathbf{3}$ and $\mathbf{4}$ were determined by comparing the $t_{R}$ of their trimethylsilyl-1-cysteine derivatives D-glucose $\left(t_{R}=21.22 \mathrm{~min}\right)$ and D-galactose $\left(t_{R}=21.56 \mathrm{~min}\right)$ with those of authentic sugars prepared by a similar procedure.

\subsection{Cytotoxicity Assay}

The cytotoxic activity of the isolated compounds against SMMC-7712, Bel-7402, A549, H460, and K562 human cancer cell lines was evaluated with the (3-(4,5-dimethylthiazol-2-yl)-2, 5-diphenyltetrazolium bromide) assay using 5-fluorouracil as a positive control. Briefly, $1 \times 10^{4}$ cells $\mathrm{mL}^{-1}$ were seeded in 96-well plates and allowed to adhere for $24 \mathrm{~h}$. Compounds 1-6 were dissolved in dimethylsulfoxide (DMSO) and 6-fold dilutions were prepared in complete medium (from 100-0.1 $\mu \mathrm{mol} \mathrm{L}{ }^{-1}$ ) for determination of inhibition rate. After incubation at $37.8^{\circ} \mathrm{C}$ for $24 \mathrm{~h}$, the supernatant was removed and DMSO $(100 \mu \mathrm{L})$ was added to each well. The inhibition rate and half-maximal inhibitory concentration $\left(\mathrm{IC}_{50}\right)$ were calculated. Compounds $\mathbf{1}-\mathbf{6}$ showed cytotoxicity against human SMMC-7712, Bel-7402, A549, H460, and K562 cell lines; the $\mathrm{IC}_{50}$ values are shown in Table 2. 
Table 2. Cytotoxicities of compounds 1-6 against five human cancer cell lines in vitro $\left(\mathrm{IC}_{50}, \mu \mathrm{M}\right)^{\mathrm{a}}$.

\begin{tabular}{cccccc}
\hline \multirow{2}{*}{ Compounds } & \multicolumn{5}{c}{ Cell Lines } \\
\cline { 2 - 6 } & SMMC-7721 & Bel-7402 & A549 & H460 & K562 \\
\hline b 5-Fu & $2.4 \pm 1.9$ & $4.3 \pm 2.1$ & $4.0 \pm 1.6$ & $1.7 \pm 2.8$ & $1.0 \pm 0.9$ \\
1 & $10.4 \pm 3.9$ & $13.3 \pm 4.6$ & $>100$ & $33.7 \pm 2.2$ & $25.5 \pm 3.5$ \\
2 & $11.2 \pm 4.4$ & $6.9 \pm 1.7$ & $14.4 \pm 2.8$ & $26.2 \pm 2.7$ & $29.3 \pm 5.3$ \\
3 & $8.6 \pm 3.4$ & $11.4 \pm 4.1$ & $>100$ & $31.4 \pm 1.7$ & $24.2 \pm 2.9$ \\
4 & $7.2 \pm 4.6$ & $4.4 \pm 1.1$ & $12.3 \pm 2.5$ & $21.8 \pm 2.1$ & $>100$ \\
5 & $7.4 \pm 5.8$ & $31.9 \pm 2.1$ & $>100$ & $34.4 \pm 3.2$ & $15.8 \pm 5.4$ \\
6 & $8.8 \pm 5.3$ & $30.3 \pm 2.4$ & $>100$ & $30.3 \pm 3.7$ & $12.3 \pm 5.5$ \\
\hline
\end{tabular}

${ }^{a} \mathrm{IC}_{50}$ values are means from three independent experiments (average $\pm \mathrm{SD}$ ) in which each compound concentration was tested in three replicate wells; ${ }^{\mathrm{b}} 5$-fluorouracil $(5-\mathrm{Fu})$ as positive control.

Supplementary Materials: The physical and spectroscopic data of compounds 5-6, and NMR and MS spectra of compounds 1-4 are available online.

Acknowledgments: The research work was financially supported by the National Natural Science Foundation of China (No. 81503195) and the Innovative Research Team in TCM Material Foundation and Key Preparation Technology (Grant No. 2012KCT-20); and the research work was supported by the Open Research Fund of Key Laboratory of Basic and New Herbal Medicament Research, Shaanxi University of Chinese Medicine (No. 2017KF02,17JS030), the Natural Science Foundation of Shaanxi Provincial Department of Education (Grant No. 16JK1219), and the Research Foundation of Shaanxi University of Chinese Medicine (Grant No. 2015PY09).

Author Contributions: H.T. conceived and designed the experiments; X.Y. and D.Z. collected the NMR data. Y.L. contributed to the acid hydrolysis and GC analysis. L.Z. and B.S. realized the evaluation of bioactivities; Z.Y. and X.S. analyzed the data; and Y.C. completed the isolation and structural elucidation and wrote the paper.

Conflicts of Interest: The authors declare no conflict of interest.

\section{References}

1. Song, X.M.; Liu, H.J. Research and Application of "Qi-Medicines" in Taibai Mountains; People's Medical Publishing House: Beijing, China, 2011.

2. Jie, W.Y.; Zeng, C.J.; Yao, Z.; Zhang, J.; Zhang, Y.; Zhang, F. Diterpene alkaloids from the roots and processed products of Aconitum pendulum. Chin. Tradit. Herbal Drugs 2010, 41, 347-351.

3. Yang, S.L.; Liu, X.K.; Wu, H.; Wang, H.B.; Qing, C. Steroidal saponins and cytoxicity of the wild edible vegetable-Smilacina atropurpurea. Steroids 2009, 74, 7-12. [CrossRef] [PubMed]

4. Zhang, Y.; Li, H.Z.; Zhang, Y.J.; Jacob, M.R.; Khan, S.I.; Li, X.C.; Yang, C.R. Atropurosides A-G, new steroidal saponins from Smilacina atropurpurea. Steroids 2006, 71, 712-719. [CrossRef] [PubMed]

5. Zhang, X.; Su, Y.F.; Chen, L.; Huang, X.; Yan, S.L.; Chai, X.; Gao, X.M. Steroidal Saponins from the Rhizomes of Smilacina henryi. Helv. Chim. Acta 2013, 96, 478-487. [CrossRef]

6. Liu, X.; Zhang, H.; Niu, X.F.; Xin, W.; Qi, L. Steroidal saponins from Smilacina japonica. Fitoterapia 2012, 83, 812-816. [CrossRef] [PubMed]

7. Zhao, S.J.; Hong, B.; Yang, L.M.; Han, Z.M. Chemical constituents and their anti-tumor activities of Smilacina japonica. Chin. Tradit. Pat. Med. 2016, 38, 332-335.

8. Zhao, S.J.; Yang, L.M.; Han, Z.M.; Han, M. Furosteroidal saponin from Smilacina japonica. China J. Chin. Mater. Med. 2011, 36, 3453-3456.

9. Li, Y.; Wang, X.; He, H.; Zhang, D.; Jiang, Y.; Yang, X.; Wang, F.; Tang, Z.; Song, X.; Yue, Z. Steroidal Saponins from the Roots and Rhizomes of Tupistra chinensis. Molecules 2015, 20, 13659-13669. [CrossRef] [PubMed]

10. Song, X.; Li, Y.; Zhang, D.; Jiang, Y.; Wang, W.; Song, B.; Tang, Z.; Cui, J.; Yue, Z. Two new spirostanol saponins from the the roots and rhizomes of Tupistra chinensis. Phytochem. Lett. 2015, 13, 6-10. [CrossRef]

11. Song, X.; Zhang, D.; He, H.; Li, Y.; Yang, X.; Deng, C.; Tang, Z.; Cui, J.; Yue, Z. Steroidal glycosides from Reineckia carnea. Fitoterapia 2015, 105, 240-245. [CrossRef] [PubMed]

12. Wang, F.; Yue, Z.; Xie, P.; Zhang, L.; Li, Z.; Song, B.; Tang, Z.; Song, X. C19-Norditerpenoid Alkaloids from Aconitum szechenyianum and Their Effects on LPS-Activated NO Production. Molecules 2016, 21, 1175. [CrossRef] [PubMed] 
13. Chai, J.; Song, X.; Wang, X.; Mei, Q.; Li, Z.; Cui, J.; Tang, Z.; Yue, Z. Two new compounds from the roots and rhizomes of Trillium tschonoskii. Phytochem. Lett. 2014, 10, 113-117. [CrossRef]

14. Zhang, D.; Wang, W.; Li, Y.; Li, Z.; Jiang, Y.; Tang, Z.; Song, X.; Yue, Z. Two new pregnane glycosides from Reineckia carnea. Phytochem. Lett. 2016, 15, 142-146. [CrossRef]

15. Yue, Z.; Qin, H.; Li, Y.; Sun, Y.; Wang, Z.; Yang, T.; Liu, L.; Wang, M.; Feng, F.; Mei, Q. Chemical constituents of the root of Jasminum giraldii. Molecules 2013, 18, 4766-4775. [CrossRef] [PubMed]

16. Agrawal, P.K. Dependence of ${ }^{1} \mathrm{H}$ NMR chemical shifts of geminal protons of glycosyloxy methylene $\left(\mathrm{H}_{2}-26\right)$ on the orientation of the 27-methyl group of furostane-type steroidal saponins. Magn. Reson. Chem. MRC 2004, 42, 990-993. [CrossRef] [PubMed]

17. Xu, J.; Feng, S.; Wang, Q.; Cao, Y.; Sun, M.; Zhang, C. Four new furostanol saponins from the rhizomes and roots of Smilax scobinicaulis and their cytotoxicity. Molecules 2014, 19, 20975-20987. [CrossRef] [PubMed]

18. Chen, H.F.; Wang, G.H.; Luo, Q.; Wang, N.L.; Yao, X.S. Two new steroidal saponins from Allium macrostemon bunge and their cytotoxity on different cancer cell lines. Molecules 2009, 14, 2246-2253. [CrossRef] [PubMed]

19. Lu, Y.; Luo, J.; Huang, X.; Kong, L. Four new steroidal glycosides from Solanum torvum and their cytotoxic activities. Steroids 2009, 74, 95. [CrossRef] [PubMed]

Sample Availability: Samples of the compounds 1-6 are available from the authors. 